\title{
Optimizing Resident Education of Ophthalmic Pathology: Qualitative Assessment of Annotated Virtual Microscopy Web Site
}

\author{
Nicole Nikolic, MD ${ }^{1,2}$ Baltazar Espiritu, MD ${ }^{1}$ Deepak P. Edward, MD ${ }^{3}$ Jay Perlman, MD, PhD ${ }^{1,4}$ \\ ${ }^{1}$ Department of Ophthalmology, Loyola University Chicago, \\ Chicago, Illinois \\ 2 Department of Ophthalmology and Visual Sciences, University of \\ Chicago, Chicago, Illinois \\ ${ }^{3}$ Wilmer Eye Institute, Johns Hopkins University, Baltimore, Maryland \\ ${ }^{4}$ Hines Veterans Affairs Hospital, Hines, Illinois \\ Address for correspondence Nicole Nikolic, MD, Department of \\ Ophthalmology and Visual Sciences, University of Chicago Medicine, \\ 5841 S. Maryland Avenue, MC 2114, Chicago, IL 60637 \\ (e-mail: nicole.nikolic@uchospitals.edu). \\ J Clin Acad Ophthalmol 2016;8:e39-e41.
}

\begin{abstract}
Keywords

- resident education

- pathology

- virtual microscopy

Introduction Virtual microscopy is an exciting new modality that is being integrated into medical education. A nationwide collaborative effort between eight leading ophthalmology programs was undertaken to compile an extensive array of ophthalmic pathology slides to host online. To facilitate self-directed learning and assessment, Web site development included "hidden" interactive annotations, accessible only on demand, of relevant pathological findings.

Methods A small subgroup of 24 ophthalmology residents currently matriculated in their second, third, or fourth postgraduate year across eight institutions independently evaluated the Web site. Educational effectiveness was assessed by anonymous survey. Features qualitatively considered included (1) level of postgraduate training, (2) prior ophthalmic pathology Web site experience, (3) perceived educational values, (4) difficulties encountered, (5) Web site utility, and (6) usefulness of a free-text comment box.

Results Two-thirds of the participating residents had prior exposure to virtual microscopy as a resource tool. Positive assessments included the use of interactive annotations by which to facilitate self-directed learning and a user-friendly platform with good-to-excellent educational value relative to other ophthalmic-related Web sites. Negative assessment included little value in problem-based learning. Suggestions for improvement included integration with pathology-related lecture series led by an ophthalmic pathologist.

Discussion The use of a comprehensive online interactive virtual library of ophthalmic pathological specimens to facilitate autodidactic medical education is supported by a small subgroup of currently matriculated ophthalmology residents across eight institutions. Integration of prereview and postreview "quizzes" addressing relevant pathological topics is anticipated to further reinforce retention of fundamental pathological concepts.
\end{abstract}

received

June 25, 2015

accepted after revision

February 12, 2016
DOI http://dx.doi.org/

10.1055/s-0036-1581110. ISSN 2379-0539.
Copyright $\odot 2016$ by Thieme Medical Publishers, Inc., 333 Seventh Avenue, New York, NY 10001, USA. Tel: +1(212) 584-4662.
License terms

(c) (1) $\ominus$ (\$) 
Virtual microscopy has revolutionized the field and teaching of pathology by integrating the virtual feel of light microscopy with digital scans of pathology slides. This has allowed for digitizing stores of pathology tissue slides for online storage and widespread access via private computer networks or the Internet. ${ }^{1-4}$ Microscopic images are uploaded online for use by anyone from any location, or they can be integrated within an interactive lecture where a pathologist can lead the discussion and direct attention to specific findings on the virtual image. These images can then be used in conjunction with image viewing software to navigate the image, zoom in or out, and add annotations. ${ }^{1,2}$

We have developed an ophthalmic pathology virtual microscopy Web site under the auspices of the American Association of Ophthalmic Pathologists (AAOOP) with the collaborative effort of several ophthalmic pathologists. Using this site, ophthalmology residents are trained on ophthalmic pathology by way of a comprehensive virtual microscopy Web site (http://path. bnbdev.com/index1a.htm), open for use by any member of the public domain who wishes to utilize the resource, without need for either compensation or registration. To foster more active learning, the microscopy slides have hidden annotations, which are invisible without use of the mouse over the area of interest. The use of a "self-test" feature with interactive annotations visible only when hovering over a point of interest on screen in virtual microscopy for resident education is a unique teaching tool, which we implemented to provide a more cohesive and effective learning experience. The purpose of this study was to obtain feedback on the educative value of the Web site for ophthalmic pathology.

\section{Materials and Methods}

\section{Virtual Microscopy Web Site at Loyola University Chicago School of Medicine}

To create an Ophthalmic Pathology Collaborative and Educational Resource (OPCER), a collaboration was forged between several leading institutions: Johns Hopkins, Duke University, University of Iowa, Northwestern University, the New York Ear and Eye Infirmary, Rush University Medical Center, University of Miami, and Loyola University Chicago.

Glass slides were collected from the ophthalmic pathology collections of the Ophthalmic Pathologists from the previously mentioned academic institutions. The slides were de-identified and then converted into digital images via the Aperio slide scanner. These digital images were then stored as Zoomify images and hosted online by the Department of Ophthalmology of Loyola University Chicago at http://path.bnbdev.com/index1a. $\mathrm{htm}$. The images are organized on the Web site by the following categories: normal, eyelid, conjunctiva, cornea, glaucoma, retina, uveal tract, nasolacrimal sac, orbit, infection, and inflammation. Annotations were made first by identifying points of interest in the image that were relevant toward making an accurate diagnosis. Once identified, a new label was created, with both a description of the findings and an appropriate icon pointing out the structure or defect. These labels were then edited to be visible only when the cursor driven by the individual hovered over the specific point of interest in the current field of view.

\section{Participants}

The Web site was evaluated by 24 ophthalmology residents who responded to the survey after it was sent out to eight ophthalmology programs. Of this group, seven were in their second postgraduate year, eight in their third postgraduate year, and nine in their fourth and final postgraduate year.

\section{Web Site Evaluation}

An online evaluation was conducted to assess the residents' impressions of the updated virtual microscopy site. The evaluation was a formal online survey composed of seven questions devised by the aforementioned corroborators of the study. Elicited information included the following: their level of postgraduate training, prior experience with virtual microscopy, prior experience with ophthalmic pathology Web sites, ease of Web site navigation, value of virtual microscopy relative to other pathology Web sites, the perceived educational value of our ophthalmic pathology virtual microscopy site, and whether they prefer the addition of annotations on the slides. An additional free-text comment box was also included in the survey for the residents to leave feedback, for consideration into future updates to the Web site.

\section{Results}

At the time of the survey, the majority of residents (67\%) had prior experience with using virtual microscopy Web sites, and $62 \%$ reported previous use of an ophthalmic pathology Web site. Junior residents were more likely to have had prior experience with virtual microscopy, with $71 \%$ of PGY-2 residents having prior experience compared with $62 \%$ of PGY-3 and $67 \%$ of PGY-4 responders. Interestingly, the group with the highest rate of previous exposure to virtual microscopy also reported the lowest reported use of ophthalmic pathology Web sites, with $43 \%$ having used the resource tool, compared with the resident average of $63 \%$.

With respect to positive feedback regarding the Web site, the site was overwhelmingly described as being user friendly (96\%), of good-to-excellent educational value (96\%), and was overall more valuable relative to other ophthalmic-related Web sites (68\%). The residents were also unanimous in finding the annotations on the various slides useful. As illustrated in - Fig. 1, the residents found the most utility of specific functions of the Web site with respect to resident education, in using the site for self-directed learning (83\%), in preparation for ophthalmology board exams (71\%), and for use during lectures led by an ophthalmic pathologist (79\%). The residents also appear to find little utility in using the Web site for problem-based learning (i.e., interactive lectures with clinical scenarios incorporating relevant histopathology), with only $29 \%$ believing it to be useful.

Encouragingly, the residents unanimously found the Web site user friendly. The residents were also unified in believing the educational value of our Web site to be good to excellent, and $83 \%$ of them also felt our virtual microscopy site was more beneficial compared with other ophthalmic pathology Web sites they have previously used. 
Which of the following do you feel the website is useful for? (Check all that apply.)

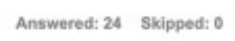

Answered: 24 skipped: 0

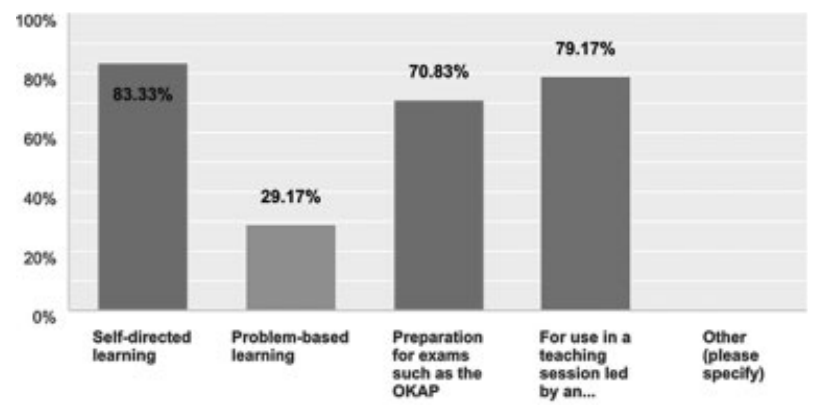

Fig. 1 Matriculated ophthalmology residents' responses for the utility of virtual microscopy with respect to various aspects of their education. Most supported was the use of the technology toward selfdirected learning and integrated into lecture series led by an ophthalmic pathologist.

\section{Discussion}

In all, we found that our addition of hidden annotations was a success with respect to the residents' unanimous agreement on the perceived utility of the function to the Web site. We also found that of the residents without previous use of virtual microscopy, all had indicated strong educational value and ease of use of our Web site. In regard to the educational value of the Web site, it was interesting to find that those without prior virtual microscopy use found our Web site to be more useful compared with other ophthalmic pathology Web sites. We offer that perhaps the reason for this was poor incorporation of the technology for those with prior experience.

While our study was limited by our sample size among responding residents, we believe the trends found in our survey are in line with general sentiments with our Web site and virtual microscopy technology overall. In the future, we plan to further the utility of the Web site with the addition of quizzes written in a fashion similar to pathology questions that appear on ophthalmology board examinations. These questions will focus on frequently asked topics and will have accompanying pictures from our collection of slides on our Web site, which can also be used to reinforce concepts for selfdirected learning.

Given our findings, we highly encourage ophthalmology residents to incorporate this resource alongside their reading for self-directed learning, especially during study in preparation for their board examinations, as residents often find pathology to be one of the most difficult topics. Additionally, as an overwhelmingly majority of residents believed the Web site would be a useful adjunct to ophthalmic pathology lectures, it is our hope that our Web site is incorporated into programs nationwide to further enhance resident education in ophthalmology. Interestingly, very few residents felt that the use of virtual microscopy would be useful in problem-based learning. However, to acquire the particular fund of knowledge, that is, ophthalmic pathology, one focuses on refining pattern-based recognition-which is often honed by the method of study du jour, computer-based question banks written in the style of board exams.

Acknowledgments

We would like to thank Drs. Paul Bryar, Thomas Cummings, Richard Grostern, Tatyana Milman, Sander Dubovy, and Nasreen Syed for their contributions to our Web site.

\section{References}

1 McCullough B, Ying X, Monticello T, Bonnefoi M. Digital microscopy imaging and new approaches in toxicologic pathology. Toxicol Pathol 2004;32(Suppl 2):49-58

2 Pantanowitz L, Szymas J, Yagi Y, Wilbur D. Whole slide imaging for educational purposes. J Pathol Inform 2012;3:46

3 Nelson D, Ziv A, Bandali KS. Going glass to digital: virtual microscopy as a simulation-based revolution in pathology and laboratory science. J Clin Pathol 2012;65(10):877-881

4 Sander B, Golas MM. HistoViewer: an interactive e-learning platform facilitating group and peer group learning. Anat Sci Educ 2013;6(3):182-190 